\title{
PRIAPISMO ARTERIAL DE ALTO FLUJO SECUNDARIO A FISTULA ARTERIO-CAVERNOSA BILATERAL POST-TRAUMÁTICA. EMBOLIZACIÓN SUPRASELECTIVA CON COÁGULO AUTÓLOGO
}

\author{
E. FERNÁNDEZ ROSADO, A. BLANCO DIEZ, A. BARBAGELATA LÓPEZ, L. ÁlVAREZ \\ CASTELO, A. LANCINA MARTÍN, J.I. CAO GONZÁLEZ*, S. NOVAS CASTRO, M. RUIBAL \\ MOLDES, V. CHANTADA ABAL, M. GONZÁLEZ MARTÍN
}

Servicio de Urología. *Servicio de Radiología Intervencionista. Hospital Universitario Juan Canalejo. La Coruña.

Actas Urol Esp. 28 (2): 152-156, 2004

\section{RESUMEN}

PRIAPISMO ARTERIAL DE ALTO FLUJO SECUNDARIO A FÍSTULA ARTERIO-CAVERNOSA BILATERAL POST-TRAUMÁTICA. EMBOLIZACIÓN SUPRASELECTIVA CON COÁGULO AUTÓLOGO

El priapismo arterial de alto flujo es una patología poco frecuente caracterizada por una erección parcial persistente no dolorosa. Por lo general, se asocia a un traumatismo perineal o peneano (área genitoperineal) que ha ocasionado una fístula arterio-cavernosa, raramente bilateral. Tan sólo se han publicado once casos en los que dicha fístula es bilateral. Los tratamientos empleados han sido diversos, sin una pauta común para su resolución. Presentamos el caso de un varón de 27 años con priapismo de alto flujo arterial secundario a traumatismo perineal con fístula arterio-cavernosa bilateral. Se empleó coágulo autólogo para la embolización de las fístulas con el fin de minimizar el riesgo de lesión de la función eréctil en un paciente joven. No se registraron complicaciones. La función eréctil es normal 8 meses después del tratamiento sin evidencia de fístula en ecografía de control. Consideramos que la arteriografia supraselectiva junto a la embolización con sustancias reabsorbibles proporcionan los mejores resultados, menores riesgos y complicaciones en este tipo de patología.

PALABRAS CLAVE: Priapismo. Fístula arterio-cavernosa bilateral. Alto flujo. Embolización. Coágulo autólogo.

\section{ABSTRACT}

HIGH-FLOW ARTERIAL PRIAPISM FOR POSTRAUMATIC BILATERAL ARTERIAL-CAVERNOUS FISTULA. HIGHLY SELECTIVE EMBOLIZATION USING AUTOLOGOUS BLOOD CLOT

High flow arterial priapism is rare and characterized to a prolonged non-painful partial erection. Arteriocavernous fistula, rarely bilateral, is associated with penile or perineal trauma (genitoperineal area). Review of the literature, in only eleven cases this fistula is bilateral. Diferents treatments with non common norm has been used. We report a case of a 27 years old male who presented high folw priapism secondary to bilateral postraumatic arterio-cavernous fistula. Autologous blood clot was used for fistulas embolization to minimize the risk of impotence in this young patient. No complications was registered. Eight months later the patient had normal erectile function and no fistula was detected on ultrasonography doppler. We consider arteriography with highly selective embolization using reabsorbable agents obtains the best results, the least risks and complications in this process.

KEYS WORDS: Priapism. Bilateral arterial cavernous fistula. High-flow. Embolization. Autologous blood clot. 
$\mathrm{E}$ priapismo consiste en una erección peneana anormal y mantenida, completa o no, prolongada en el tiempo, dolorosa o indolora, no reaccionada con el estímulo sexual y limitada a los cuerpos cavernosos sin afectación del cuerpo esponjoso o el glande, causando un desequilibrio hemodinámico entre los mecanismos de aporte sanguíneo arterial y drenaje venoso del pene.

El priapismo más frecuente es el de bajo flujo, veno-oclusivo, isquémico o de estasis. En éste se interrumpe el drenaje venoso sanguíneo de los cuerpos cavernosos, expandiéndose éstos al máximo, causando por ello dolor e impidiendo la entrada de más aporte sanguíneo arterial oxigenado, produciendo isquemia tisular. Potencialmente este tipo de priapismo puede ser lesivo sobre la función eréctil y por ello debe ser considerado como una urgencia inmediata.

En el priapismo de alto flujo, arterial, no isquémico, se encuentra aumentado el aporte sanguíneo arterial a los cuerpos cavernosos de modo permanente, siendo el drenaje venoso insuficiente para tal aporte, manteniéndose un estado de tumescencia o rigidez parcial no dolorosa y por lo general bien tolerada. Tiene mejor pronóstico y puede considerarse como una situación de urgencia diferida. Este tipo de priapismo tiene generalmente una historia de traumatismo perineo-escrotal (área genitoperineal) o peneano.

El diagnóstico diferencial suele ser sencillo y basado principalmente en la clínica y anamnesis del paciente, y apoyarse en la realización de una ecografía doppler peneana (flujo de estasis o flujo aumentado), gasometría sanguínea de los cuerpos cavernosos (hipoxemia en el venooclusivo de estasis), y arteriografía (método más efectivo para el diagnóstico y tratamiento mediante embolización de los priapismos arteriales de alto flujo secundarios a fístula arteriocavernosa).

Existen once casos publicados de priapismos de alto flujo arterial secundarios a fístulas arterio-lacunares bilaterales ${ }^{1}$. Nosotros presentamos un nuevo caso de fístula bilateral con completa iconografía y resuelto con éxito por la escasa frecuencia de estas fístulas bilaterales y la controversia existente respecto al tratamiento de las mismas.

\section{CASO CLÍNICO}

Varón sano de 27 años remitido a nuestro Servicio por cuadro de priapismo no doloroso de una semana de evolución, con instauración paulatina tras traumatismo perineal por caída a horcajadas en una alcantarilla sin tapa.

A la exploración presentaba priapismo parcial no completo, elástico, semirígido, no doloroso a la palpación y que permitía micciones normales. No se evidenció hematoma perineal, escrotal ni peneano (Fig. 1).

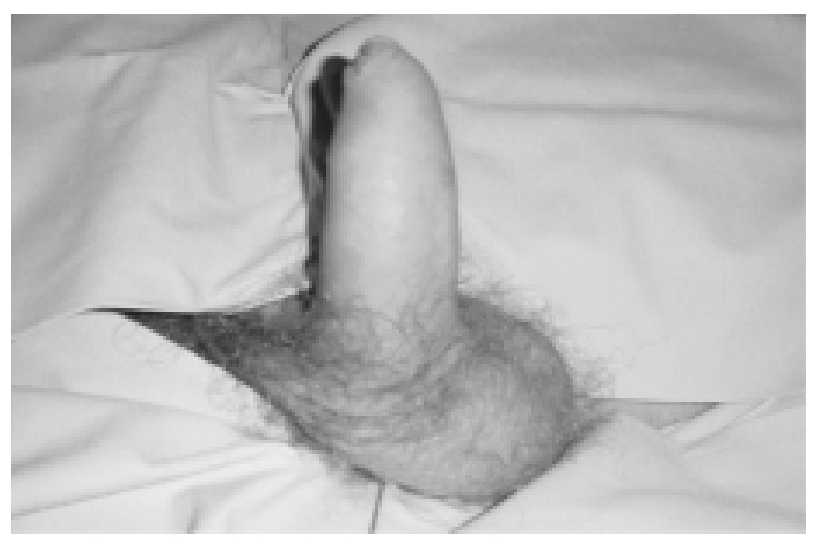

FIGURA 1. Priapismo parcial semirigido no doloroso tras una semana del traumatismo perineal tal y como fue remitido a nuestro Servicio.

La gasometría por aspiración de los cuerpos cavernosos mostró un pH normal igual que la pO2 y la pCO2, valores similares a los de la sangre arterial evidenciando la presencia de sangre altamente oxigenada, arterial, en el interior de los espacios lacunares de los cuerpos cavernosos. La ecografía doppler de los cuerpos cavernosos evidenció un aumento de flujo sanguíneo, más evidente en el lado derecho, donde se observaban grandes espacios lacunares dilatados con flujo turbulento interno de casi un centímetro de diámetro (Figs. 2A y 2B).

Se realizó arteriografía pélvica supraselectiva que mostró extravasado de contraste bilateral en ambos cuerpos cavernosos, en la base del pene evidenciando el diagnóstico de sospecha de fístula arterio-cavernosa (arterio-lacunar) bilateral. Se realizó inicialmente embolización selectiva de la fístula derecha con $2 \mathrm{ml}$ de coágulo autólogo y posteriormente a las $72 \mathrm{~h}$ y tras fracaso de esta 


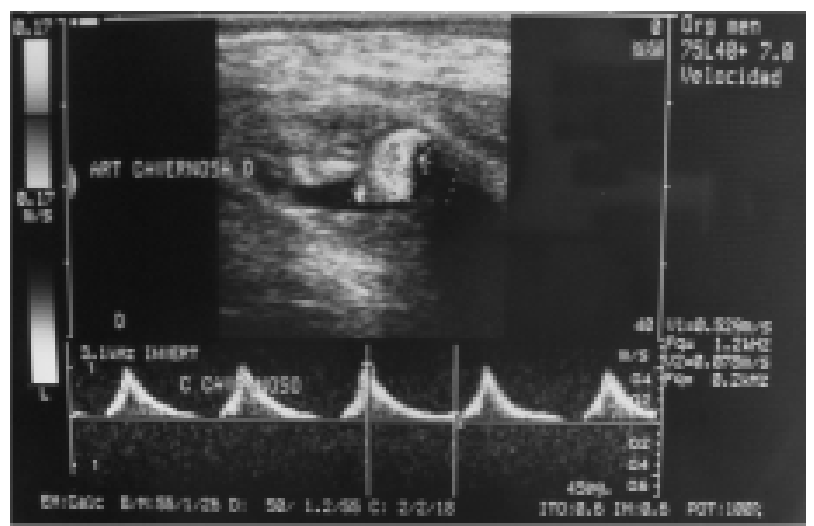

FIGURA 2A. Ecografia doppler del cuerpo cavernoso derecho en donde se evidencian espacios lacunares dilatados con alto flujo arterial y turbulencias internas.

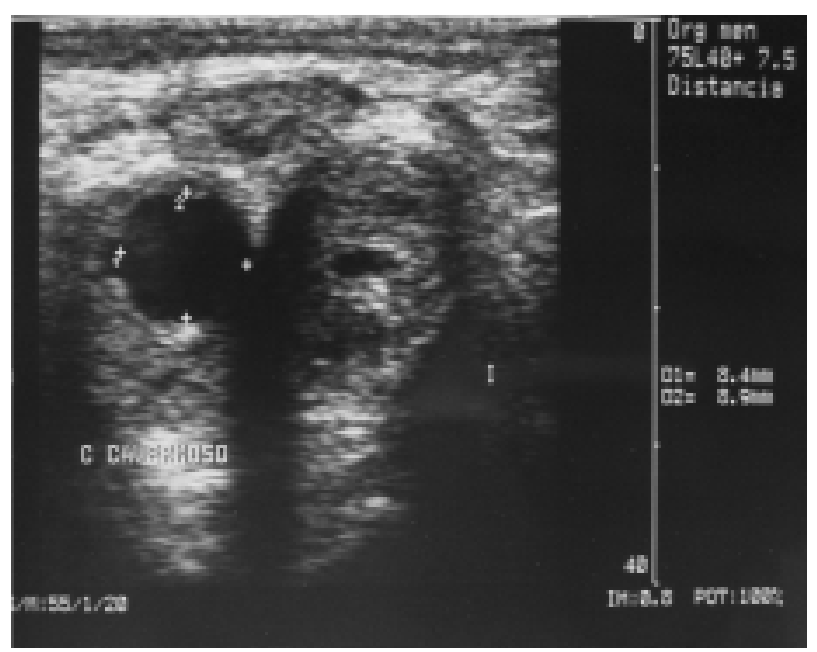

FIGURA 2B. Ecografia doppler de ambos cuerpos cavernosos que evidencia la dilatación de casi un centimetro de diámetro de un espacio lacunar dentro del cuerpo cavernoso derecho y con flujo turbulento en su interior en ambos lados.

primera maniobra, se repitió el proceso con embolización de un lado y a continuación el otro debido a la no-mejoría clínica con la embolización unilateral (Figs. 3A, 3B y 3C). El resultado final consistió en la detumescencia gradual prácticamente total a las $24 \mathrm{~h}$ (Fig. 4). No se registraron ningún tipo de complicaciones en relación con la arteriografía y la embolización. La ecografía doppler de control tras la embolización no mostró las alteraciones evidentes al inicio del proceso.

El seguimiento actual del paciente es de 8 meses con revisiones periódicas y función sexual completamente normal, tanto en las erecciones como en las detumescencias.

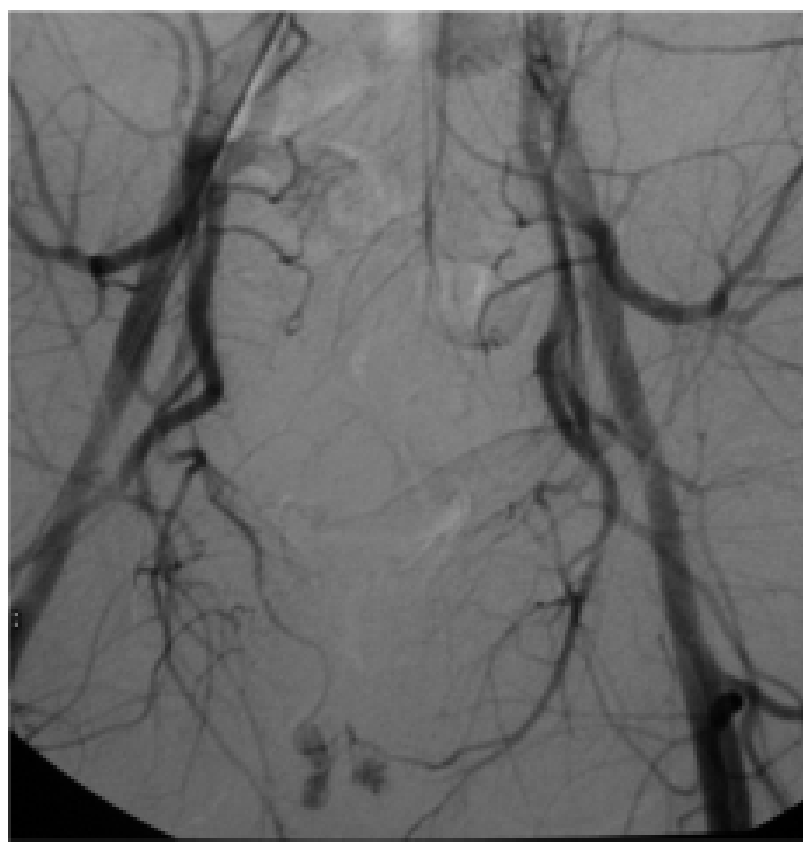

FIGURA 3A. Arteriografia en donde se evidencia la fuga de contraste bilateral en ambos cuerpos cavernosos.

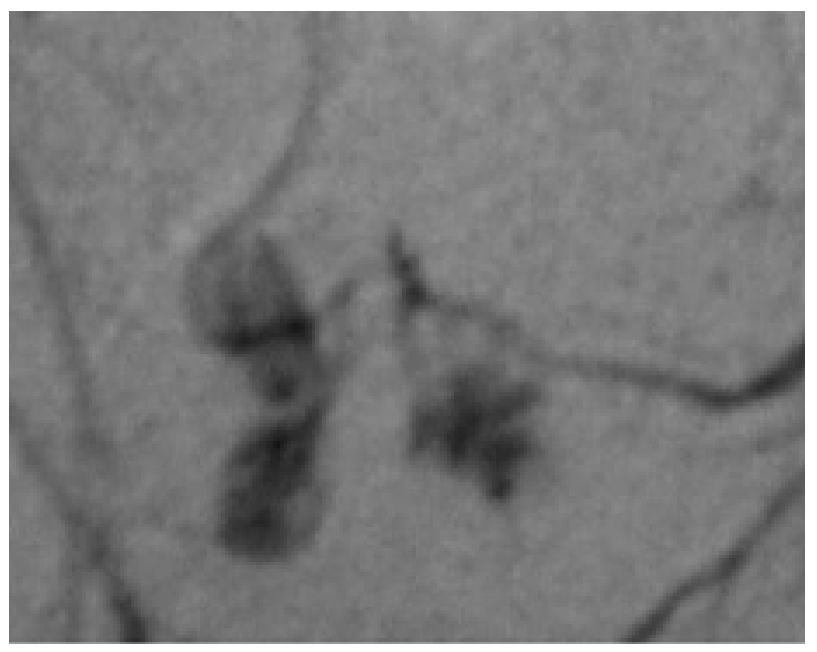

FIGURA 3B. Detalle ampliado de la fuga de contraste bilateral en la arteriografia dando lugar a la fistula arterio-cavernosa (arterio-lacunar) bilateral.

\section{DISCUSIÓN}

El priapismo arterial de alto flujo no isquémico tiene generalmente una evolución progresiva persistente y no dolorosa, pudiendo ser manejado por lo general de modo diferido a diferencia del veno-oclusivo de estasis o isquémico, que requiere un manejo urgente con el fin de evitar la lesión tisular hipóxica irreversible. 


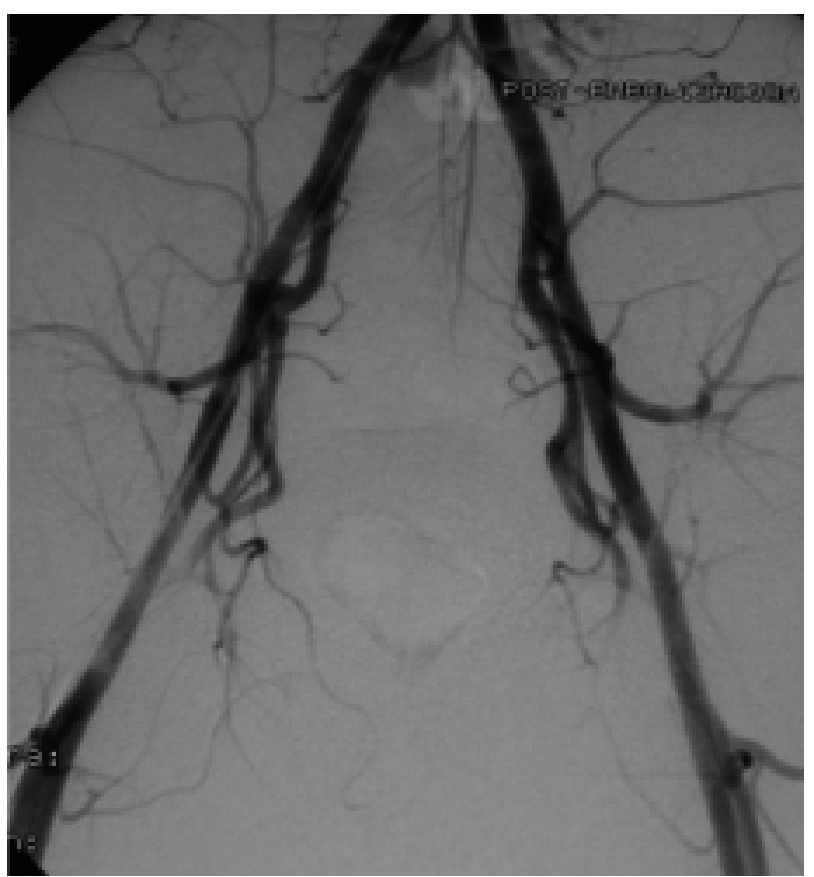

FIGURA 3C. Arteriografia tras embolización supraselectiva con $2 \mathrm{ml}$ de coágulo autólogo de ambas arterias fistulosas, muy próximo a la lesión con cese del flujo fistuloso.

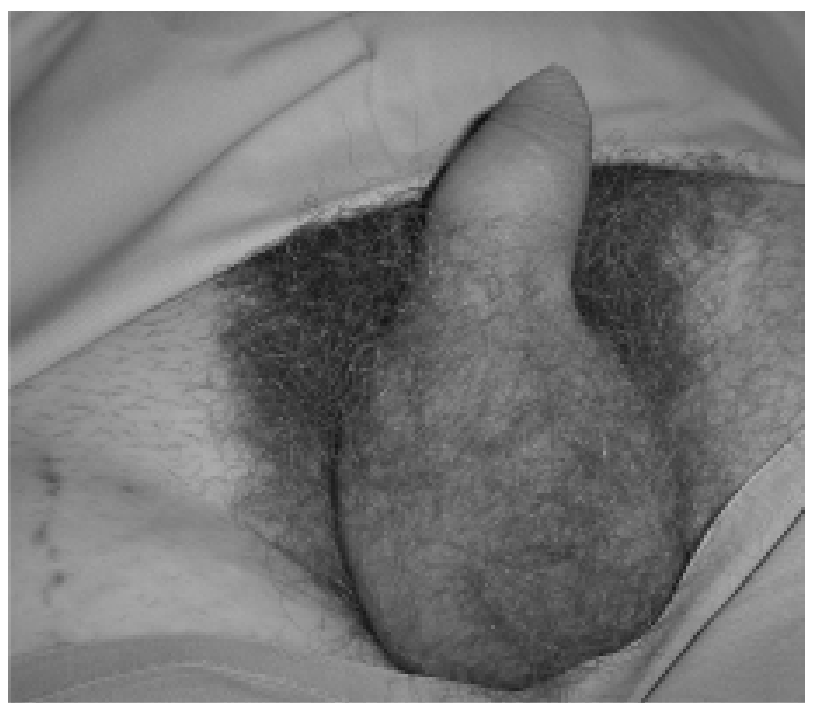

FIGURA 4. Resultado final a las 24 h de la embolización bilateral con detumescencia total.

Suele existir el antecedente traumático, pero en ocasiones éste puede acontecer varios días antes de la consulta e incluso puede no existir en casos de yatrogenia como las autoinyecciones de sustancias vasoactivas intracavernosas en el contexto terapéutico de la disfunción eréctil.

Ante la sospecha clínica de esta patología la exploración (rigidez parcial no dolorosa), la gaso- metría de los cuerpos cavernosos (patrones de $\mathrm{pH}, \mathrm{pO} 2, \mathrm{y}$ pCO2 similares a los valores normales de sangre arterial), la ecografía peneana (alto flujo y turbulencias dentro de los espacios lacunares de los cuerpos cavernosos), y la arteriografía son las principales armas diagnósticas.

Esta última es el mejor método diagnóstico y terapéutico en este grupo de priapismos, mostrando la extravasación de contraste en los espacios lacunares de los cuerpos cavernosos.

El manejo conservador inicial puede ser útil debido al bajo riesgo de isquemia tisular a diferencia de los priapismos veno-oclusivos. Por lo general dicho manejo con compresión mecánica y frío local no suele ser efectivo, al igual que el drenaje-aspirado de los cuerpos cavernosos e irrigación con drogas alfa agonistas. El tratamiento radiointervencionista se prefiere al tratamiento quirúrgico, aunque la ligadura quirúrgica de las ramas arteriales fistulosas tiene su indicación ante un fracaso repetitivo de las embolizaciones selectivas.

El tratamiento invasivo se realiza tras observar fracaso en la detumescencia con el manejo conservador. La arteriografía supraselectiva (con el fin de no dañar vasos sanos) con embolización distal máxima de la arteria fistulosa es la intervención con menos complicaciones y mejores resultados. Dicha supraselectividad disminuye además el posible riesgo de embolia a distancia por desprendimiento del material empleado ${ }^{2,3}$.

Los casos descritos bien documentados de fístulas arterio-cavernosas bilaterales son escasos en la literatura (once casos en total) ${ }^{4}$.

Diversas sustancias han sido empleadas para la embolización (reabsorbibles y no reabsorbibles) al igual que diversos procedimientos (una sesión, dos sesiones,...). En 1977 Wear et al. ${ }^{5}$ realizaron la primera embolización de una fístula arterio-lacunar con coágulo autólogo. Esta sustancia, al igual que las esponjas de gelatina reabsorbibles (spongostan, gelfoam), permiten la recanalización de la arteria embolizada una vez que se disuelven a los pocos días (tiempo suficiente para que se produzca la auto-reparación de la lesión en la pared arterial), preservando de mejor manera la función eréctil normal previa. Así, la interrupción del flujo sanguíneo de los vasos lesionados puede considerarse temporal. 
La embolización con sustancias no reabsorbibles (bucrylato, microcoil,...), pueden causar disfunción eréctil o disminución de la rigidez eréctil por falta de recanalización arterial posterior ${ }^{1}$. Las sustancias no reabsorbibles radiopacas (como los microcoils) tienen a su favor la propiedad de permitir una mayor exactitud en su posicionamiento y mejor control radiológico y seguimiento posterior debido a su radiopacidad ${ }^{6}$.

Por estas razones, se prefiere el uso de sustancias reabsorbibles a las no reabsorbibles o permanentes ya que la disolución de las sustancias embolígenas es esencial para el restablecimiento del flujo arterial normal y minimizar así el riesgo de disfunción eréctil posterior, aunque existen algunos casos publicados en los que se empleó material no reabsorbible en caso de fístulas bilaterales ${ }^{6}$.

Se prefiere el uso de coágulo autólogo sobre las otras sustancias reabsorbibles por su fácil obtención, manejo, coste y buenos resultados. Son suficientes $3 \mathrm{ml}$ para la oclusión completa temporal de la arteria fistulosa ${ }^{7}$.

Por lo general, se prefiere iniciar el proceso con la embolización de un solo lado, ya que de este modo se minimiza el riesgo de lesión de la función eréctil, isquemia o incluso de gangrena si se realiza la embolización de manera excesivamente agresiva, poco selectiva y de modo bilateral. De todos modos es obvio que la embolización bilateral en un solo tiempo evita la repetición de un proceso intervencionista agresivo y no falto de posibles complicaciones aunque éstas suelen ser raras.

En nuestro caso la embolización de un lado único con material reabsorbible (coágulo autólogo) fracasó inicialmente y se realizó en un segundo tiempo una embolización bilateral con material reabsorbible para evitar una tercera intervención, con el resultado de una detumescencia prácticamente total a las $24 \mathrm{~h}$.

La recanalización de las arterias lesionadas es un factor especialmente importante en las fístulas bilaterales y por ello la importancia del uso de material embolígeno reabsorbible, sobre todo en pacientes jóvenes. No se aconseja el uso de material no reabsorbible de modo bilateral por el riesgo de complicaciones y posible afectación sobre la función eréctil posterior.

En conclusión las fístulas arterio-cavernosas bilaterales con priapismo secundario pueden solucionarse con éxito, seguridad, efectividad y buenos resultados con embolización supraselectiva con material reabsorbible, prefiriendo el uso de coágulo autólogo al resto de los materiales sintéticos.

\section{REFERENCIAS}

1. LANGENHUIJSEN JF, REISMAN Y, REEKERS JA, DE REIJKE TH M.: Highly selective embolization of bilateral cavernous arteries for post-traumatic penile arterial priapism. Int $J$ Impot Res $2001 \mathrm{dec} ; 13$ (6): 354-356.

2. COLOMBO F et al.: Arterial embolization in the treatment of post-traumatic priapism. Ann Urol 1999; 33: $210-218$.

3. KERLAN RK, GORDON RL, LABERGE JM, RING EJ.: Superselective microcoil embolization in the management of high-flow priapism. $J$ Vasc Interv Radiol 1998; 9: 85-89.

4. DE PABLO CÁRDENAS A, JIMÉNEZ ARISTU JI, LOZANO URUÑUELA $F$ et al.: High flow priapism secondary to bilateral arterial-lacunar fistula. Arch Esp Urol 2002; 55 (7): 821-825.

5. WEAR JB, CRUMMY AB, MUNSON BO.: A new approach to the treatment of priapism. J Urol 1977; 117: 252-254.

6. GUJRAL S, MACDONAGH RP, CAVANAGH PM.: Bilateral superselective arterial microcil embolisation in delayed post-traumatic high flow priapism. Postgrad Med J 2001; 77: 193-194.

7. LAZINGER M, BECKMANN CF, COSSI A, ROTH RA.: Selective embolization of bilateral arterial cavernous fistulas for post-traumatic penile arterial priapism. Cardiovasc Intervent Radiol 1996; 19: 281-284.

Dr. E. Fernández Rosado

C/ Ronda de Outeiro, 133 - 2º Izda

15007 La Coruña

(Trabajo recibido el 6 febrero de 2003) 